\title{
Cranial Morphology, Y-Chromosome Variation and the History of Human Peopling
}

\author{
S. Di Marco', G. D'Amore \\ ${ }^{1}$ Laboratorio di Archeoantropologia della Sopr. per i Beni Archeologici della Toscana, Scandicci, Firenze. \\ E-mail: gdamore@unifi.it
}

KEYWORDS: NRY haplogroups, craniometric traits, recent human evolution.

\section{Introduction}

Knowledge of human biological history has recently benefited from information derived from non recombinant DNA markers such mtDNA and NRY.These formidable new tools are useful for reconstructing crucial events and processes of human settlement. However, reconstruction of human population history needs to benefit from a multidisciplinary approach because genetic analyses based on inferences from contemporary populations are difficult to interpret. It is best to integrate a wide array of information including osteoanthropological, archaeological, geological, biochemical, climatic and linguistic data. Here we analyzed the main morphological cranial features that distinguish major, present day human groups in prehistoric samples dated from 160,000 to 5,000 years B.P.The aim was to use the patterns of morphological differences to formulate hypotheses concerning continuity/discontinuity, migration and admixture among populations in different areas of the world.

\section{Materials and Methods}

Data for 32 cranial measurements (defined in Howells, 1989) were collected for 1721 skulls from 45 recent population samples, and for 114 skulls from prehistoric population samples (older than 5000 years before the present). Since the analyzed samples were mainly males, genetic relationship among recent populations used as reference samples were based on the actual distribution of NRY haplogroups (hgs) frequencies ( $Y$ Chromosome Consortium, 2011). Recent cranial samples were subdivided among 18 major groups according to their genetic relationships as inferred by NRY hgs. Prehistoric samples were subdivided into 13 groups according to their geographical provenance, absolute chronology and archaeological-cultural context. Data were taken from literature, particularly from Howells (1989) for recent groups and from several sources for prehistoric groups. We present original data for Sardinians, Sicilians, other Italians, and Fuegians. Average values for the 32 measurements were calculated for all the $18+13$ groups, which were Q-mode standardized (Jungers et al., 1995) in order to separate size and shape information. Then, measurements which were able to best characterize, distinguish and differentiate between the 18 recent groups were selected. The selection criterion consisted in defining a threshold value that, by inspection of all the average values for a given measurement, was systematically exceeded by all the samples included in a group.

The distinctive traits were considered "developed" (if the average value was above an "upper" threshold value) or "reduced" (if the average value was below a "lower" threshold value). The threshold values obtained for the recent groups were then analyzed in the prehistoric groups, to detect the presence or absence of the same characterising features and of their polarity of their expression.

\section{Results and Discussion}

NRY hg frequency distribution patterns appear to support our preliminary subdivision of recent populations into 18 major groups. Analysis of distinctive traits for the recent groups produced 14 (out of the original 32) measurements that clearly characterized some groups. Sub-Saharan Africans possessed 8 distinctive traits, the highest number for all groups.

This finding is noteworthy because of the well-known ancient origin of the modern human line in Africa. Some of the African traits were variously present in other groups, such as Australians, Papua-Melanesians, and South Asians. The African condition "wide nasal opening" was present also in the Siberian Buryats and Yakuts, and in some Southeast Asian samples. Therefore, such a condition appears to be a non-adaptive feature, because it is present not only in many equatorial peoples but also in other populations living in cold climates, all possessing high frequency of the NRY $C$ hg. The 5 traits which were distinctive for the Australians were variously present both in other Oceanic groups (Papua-Melanesians, Micronesians and Polynesians) and in other populations possibly descending from ancient migrations:Ainu and Andamanese (high frequency of $\mathrm{D} \mathrm{hg}$ ), and South Asians (distinctive frequencies of $F, H, L$ hgs). The various East Asian groups shared a total of 7 distinctive traits, whereas European 
and non European "Caucasoids" and Amerindian groups showed a smaller number of distinctive traits. After the analysis of the occurrence of these distinctive traits in the prehistoric groups, the following main findings can be synthesized:

I. Several distinctive Australian traits persist in all the prehistoric groups up to the early Holocene and, in the case of Fuegians, even more recently. The occurrence of these traits decreases strongly only starting with the Neolithic samples. Their sporadic presence outside of the Australia may be interpreted as the retention of "archaic" features possibly linked to heavy mastication in hunter-gatherer populations.

2. The distinctive African traits occur also in other prehistoric groups, even if less frequently than the Australian traits. They are frequent in the oldest early anatomically modern $H$. sapiens from Africa and the Near East, but presented a lower occurrence in latest Pleistocene and early/middle Holocene Sub-Saharan Africans, maybe because of a (genetically hypothesized) "back-migration" of "Caucasoid" peoples from Western Asia to Eastern Africa more than 20,000 years ago, well before the recent Bantu expansion.

3. The morphological patterns for the European prehistoric groups support the notion that both autochthonous Paleo-Mesolithic Europeans and Near East Neolithic migrant peoples contributed to the actual gene pool, not necessarily with the same proportions inferred by genetic studies.A North African/South Asian distinctive trait, "narrow bizygomatic breadth" occurs today in Sardinians; this condition may be interpreted as the morphological marker of a Levantine Neolithic diffusion, but it is also present in an Iberian Mesolithic sample, leaving open the possibility of a Western European origin from the hypothetical Franco-Cantabrian Glacial Refugium.
4. East Asian prehistoric samples seem to suggest a discontinuity between Upper Palaeolithic and Neolithic in China, but otherwise a continuity in Japan alongside the Jomon culture. Chinese Neolithic peoples might have had an exogenous origin, coming at least partially from South Asia. Many of the distinctive traits of both Northern and Southern Far Eastern Asians should originate in a more recent (post-Neolithic) age.

5. Present-day Australo-Melanesian populations seem to possess deep roots in Africa. A gradual trend with small morphological changes is observable in the succession: "early African and Near East H. sapiens - prehistoric Australians - recent Australo-Melanesians",.

6. Peopling of the Americas (the last continent to be colonized) leaves opened many questions. Our study suggests a biological contribution of the so-called Paleoamericans of the late Pleistocene and early Holocene (between 12,500 and 6,500 years ago) to some late Holocene populations such the Fuegians. The general morphology of the other recent Amerindian samples, on the contrary, supports their origin from different time-successive migratory waves coming from Eastern and Arctic Asia during early and middle Holocene.

\section{References}

Howells W.W., 1989. Skull Shapes and the Map. Craniometric Analysis in the Dispersion of Modern Homo. Papers of the Peabody Museum of Archaeology and Ethnology, Harvard University, Cambridge, MA, 79: 189 pp.

Jungers W.L., Falsetti A.B., Wall C.E., 1995. Shape, relative size, and size-adjustments in morphometrics. Yearb. Phys. Anthrop. 38: 137-161.

Y Chromosome Consortium, 2011. International Society of Genetic Genealogy, http://www.isogg.org/tree/index11.html. 